\title{
In vitro analysis of hepatic stellate cell activation influenced by transmembrane 6 superfamily 2 polymorphism
}

\author{
SONGYAO LIU ${ }^{1,2}$, EISUKE MURAKAMI ${ }^{1,2}$, TAKASHI NAKAHARA ${ }^{1,2}$, KAZUKI OHYA $^{1,2}$, \\ YUJI TERAOKA $^{1,2}$, GRACE NASWA MAKOKHA $^{1,2}$, TAKURO UCHIDA $^{1,2}$, KEI MORIO $^{1,2}$, \\ HATSUE FUJINO $^{1,2}$, ATSUSHI ONO ${ }^{1,2}$, MASAMI YAMAUCHI ${ }^{1,2}$, TOMOKAZU KAWAOKA ${ }^{1,2}$, \\ DAIKI MIKI ${ }^{1,2}$, MASATAKA TSUGE ${ }^{1-3}$, AKIRA HIRAMATSU $^{1,2}$, HIROMI ABE-CHAYAMA $^{1,2,4}$, \\ C. NELSON HAYES ${ }^{1,2}$, MICHIO IMAMURA ${ }^{1,2}$, HIROSHI AIKATA ${ }^{1,2}$ and KAZUAKI CHAYAMA ${ }^{1,2,5}$

\begin{abstract}
${ }^{1}$ Department of Gastroenterology and Metabolism, Graduate School of Life Sciences, Institute of Biomedical and Health Sciences; ${ }^{2}$ Liver Research Project Center, Hiroshima University, Hiroshima $734-8551 ;{ }^{3}$ Natural Science Center for Basic Research and Development, Hiroshima University, Hiroshima 739-0046; ${ }^{4}$ Center for Medical Specialist Graduate Education and Research, Institute of Biomedical and Health Sciences, Hiroshima University, Hiroshima 734-8551; ${ }^{5}$ Institute of Physical and Chemical Research (RIKEN) Center for Integrative Medical Sciences, Yokohama 230-0045, Japan
\end{abstract}

Received May 21, 2020; Accepted September 15, 2020

DOI: $10.3892 / \mathrm{mmr} .2020 .11654$

\begin{abstract}
Non-alcoholic steatohepatitis (NASH) may progress via liver fibrosis along with hepatic stellate cell (HSC) activation. A single nucleotide polymorphism (SNP; rs58542926) located in transmembrane 6 superfamily 2 (TM6SF2) has been reported to be significantly associated with fibrosis in patients with NASH, but the precise mechanism is still unknown. The present study aimed to explore the role of TM6SF2 in HSC activation in vitro. Plasmids producing TM6SF2 wild-type (WT) and mutant type (MT) containing E167K amino acid substitution were constructed, and the activation of LX-2 cells was analyzed by overexpressing or knocking down TM6SF2 under transforming growth factor $\beta 1$ (TGF $\beta$ ) treatment. Intracellular $\alpha$-smooth muscle actin ( $\alpha$ SMA) expression in LX-2 cells was significantly repressed by TM6SF2-WT overexpression and increased by TM6SF2 knockdown. Following treatment with TGF $\beta$, $\alpha \mathrm{SMA}$ expression was restored in TM6SF2-WT overexpressed LX-2 cells and was enhanced in TM6SF2 knocked-down LX-2 cells. Comparing aSMA expression under TM6SF2-WT or -MT overexpression,
\end{abstract}

Correspondence to: Professor Kazuaki Chayama, Department of Gastroenterology and Metabolism, Graduate School of Life Sciences, Institute of Biomedical and Health Sciences, Hiroshima University, 1-2-3 Kasumi, Minami-ku, Hiroshima 734-8551, Japan E-mail: chayama@hiroshima-u.ac.jp

Abbreviations: HCS, hepatic stellate cell; TM6SF2, transmembrane 6 superfamily 2; TGF $\beta 1$, transforming growth factor $\beta 1$; $\alpha \mathrm{SMA}$, $\alpha$-smooth muscle actin; SNP, single nucleotide polymorphism

Key words: hepatic stellate cell, liver fibrosis, non-alcoholic steatohepatitis, single nucleotide polymorphism, tm6sf2 expression of $\alpha \mathrm{SMA}$ in TM6SF2-MT overexpressed cells was higher than that in TM6SF2-WT cells and was further enhanced by TGF $\beta$ treatment. The present study demonstrated that intracellular $\alpha$ SMA expression in HCS was negatively regulated by TM6SF2 while the E167K substitution released this negative regulation and led to enhanced HSC activation by TGF $\beta$. These results suggest that the SNP in TM6SF2 may relate to sensitivity of HSC activation.

\section{Introduction}

Non-alcoholic fatty liver disease (NAFLD) is becoming one of the most prevalent chronic liver diseases in modern countries, increasing rapidly as a result of recent upward trends in obesity and life-style changes (1). A subset of NAFLD patients go on to develop non-alcoholic steatohepatitis (NASH) by progression of steatosis and necro-inflammatory changes in the liver, leading to an increase in the incidence of hepatocellular carcinoma (2). Mortality in NAFLD patients has been reported to be independently associated with the stage of liver fibrosis (3), and it is important to prevent the progression of liver fibrosis in NAFLD patients. Recently, several drugs have been developed and have entered phase 2 or 3 clinical trials, but no effective drugs against NAFLD are yet available. Therefore, it is important to clarify the mechanism of liver fibrosis in NAFLD in order to identify therapeutic targets.

To identify clinical factors associated with the progression of liver fibrosis in NAFLD patients, several genome wide association studies (GWAS) have recently been performed worldwide. A single nucleotide polymorphism (SNP) at rs738409 in patatin-like phospholipase domain containing 3 (PNPLA3) was identified as having strong associations with prevalence and disease progression in NAFLD and NASH (4-7). A SNP in transmembrane 6 superfamily 2 (TM6SF2) was also identified as a potential contributor to NAFLD pathogenesis $(8,9)$. The SNP rs58542926 in TM6SF2 is significantly associated with 
incidence of NAFLD and with fibrosis stage (10-13). TM6SF2 protein is highly expressed in the small intestine and liver and plays a role in lipid synthesis and secretion of triglyceride-rich lipoproteins in the liver (14-19). TM6SF2 rs58542926 (C>T), a coding SNP that causes an amino acid substitution at codon 167 (E167K), is considered to lead to a loss of function and to accelerate hepatic steatosis (20). However, although lipids are metabolized in hepatocytes and may accumulate in these cells, liver fibrosis is strongly associated with hepatic stellate cells (HSCs) (21). The influence of the coding SNP in TM6SF2 on the function of HSCs has not been clarified.

HSCs are normally activated in response to stimulation by inflammatory cytokines, such as transforming growth factor beta 1 (TGF 1 1), and by pathogen-associated molecular patterns, such as lipopolysaccharides (21). Activated HSCs transform into myofibroblasts, and alpha-smooth muscle actin ( $\alpha$ SMA) expression is upregulated in the transformed myofibroblasts $(21,22)$. Activation of HSCs leads to secretion of extra-cellular matrix proteins such as collagen type 1 into the sinusoids, resulting in collagen accumulation and progression of liver fibrosis (21-23). Although the impacts of genetic factors on clinical features of NAFLD and hepatocyte functions have been analyzed, the impacts of genetic factors on HSCs have not been examined. In the present study, we explored the role of TM6SF2 SNP rs58542926 in liver fibrosis using an in vitro activated HSC model.

\section{Materials and methods}

Construction of TM6SF2 expression plasmids. Human TM6SF2 mRNA was amplified from LX-2 cells and cloned into p3xFLAG-CMV-10 vector (Sigma-Aldrich). The cloned plasmid containing the wild-type CC genotype at rs58542926 in TM6SF2 gene was designated as p3FLAG/TM6SF2-WT. Subsequently, a modified plasmid, designated as p3FLAG/TM6SF2-MT, was generated by introducing a C-to-T point mutation at rs58542926 in TM6SF2 to create an amino acid substitution [glutamic acid (E) to lysine (K)] in the TM6SF2 gene using the QuikChange Site-Directed Mutagenesis kit (Agilent Technologies).

Cell culture. LX-2 cells from a human hepatic stellate cell line, which were provided by Dr Mutsumi Miyauchi (Hiroshima University, Hiroshima, Japan), were grown in Dulbecco's modified Eagle's medium (DMEM) supplemented with $10 \%$ (v/v) fetal bovine serum at $37^{\circ} \mathrm{C}$ and under $5 \% \mathrm{CO}_{2}$. Mycoplasma testing was done before and after the experiment.

Each TM6SF2 expression plasmid was transiently transfected into LX-2 cells by FuGENE HD Transfection reagent (Promega) in accordance with the instructions supplied by the manufacturer. Twenty-four hours after transfection, transfected cells were stimulated with $10 \mathrm{ng} / \mathrm{ml}$ of TGF $\beta 1$ for $48 \mathrm{~h}$, and then the cells were harvested and stored at $-80^{\circ} \mathrm{C}$ until use.

Quantification of mRNA expression level. Total RNA was extracted from collected LX-2 cells using RNeasy Mini kit (Qiagen) and reverse-transcribed using ReverTra Ace (Toyobo Co., Ltd.) and random primer in accordance with the instructions supplied by the manufacturer. $\alpha$ SMA or TM6SF 2 mRNA levels were quantified from the resulting cDNA by quantitative PCR using the 7300 Real-Time PCR System (Applied Biosystems), with the expression of GAPDH serving as a control. Expression levels were compared using the Wilcoxon signed-rank test. Amplification was performed in a $25 \mu \mathrm{l}$ reaction mixture containing $12.5 \mu 1$ SYBR-Green PCR Master Mix (Applied Biosystems), 5 pmol of forward primer, 5 pmol of reverse primer, and $1 \mu \mathrm{l}$ of cDNA solution. After incubation for $2 \mathrm{~min}$ at $50^{\circ} \mathrm{C}$, the sample was denatured for $10 \mathrm{~min}$ at $95^{\circ} \mathrm{C}$, followed by a PCR cycling program consisting of 40 cycles of $15 \mathrm{sec}$ at $95^{\circ} \mathrm{C}, 30 \mathrm{sec}$ at $55^{\circ} \mathrm{C}$ and $60 \mathrm{sec}$ at $60^{\circ} \mathrm{C}$. The following primer sequences were used: $\alpha$ SMA; 5 '-CTC ATTTTCAAAGTCCAGAGCTACA-3' and 5'-AGCGTGGCT ATTCCTTCGT-3', TM6SF2; 5'-TGAAGCCCACCACATAGC TG-3' and 5'-CGGTCTACAGCTTGTCCCAT-3', GAPDH; 5'-GAAGGTGAAGGTCGGAGTC-3' and 5'-GAAGATGGT GATGGGATTTC-3'.

Automated capillary western blotting. LX-2 cells, transfected with TM6SF2 expression plasmids and treated with TGF $\beta 1$, were cooled on ice and dissolved with RIPA-like buffer [50 mM Tris- $\mathrm{HCl}$ (pH 8.0), 0.1\% SDS, 1\% NP-40, $150 \mathrm{mM}$ sodium chloride, and $0.5 \%$ sodium deoxycholate] containing protease inhibitor cocktail (Sigma-Aldrich). Cell lysates were transferred onto capillary western immunoassay using Wes system (ProteinSimple). The proteins were detected with anti-TM6SF2 rabbit polyclonal antibody (Thermo Fisher Scientific, Inc.), anti- $\alpha$ SMA rabbit monoclonal antibody (Cell Signaling Technology Japan), or anti-GAPDH rabbit polyclonal antibody (Santa Cruz Biotechnology), followed by anti-rabbit immunoglobulin (GE Healthcare). Signal intensities were quantified using Compass software (ProteinSimple) and were corrected by GAPDH and analyzed by Mann-Whitney U test.

Knockdown of TM6SF 2 by siRNA treatment. TM6SF2 siRNA was designed by siDirect (http://sidirect2.rnai.jp) using the TM6SF2 mRNA sequence (NM_001001524) as a reference. The designed siRNA sequence was as follows: 5'-AAAAUU CCGGUAUCUCUUCCU-3', 5'-GAAGAGAUACCGGAA UUUUGG-3'. Prepared siRNAs were transfected into LX-2 cells by electroporation using the Neon transfection system (Thermo Fisher Scientific, Inc.) at $1,100 \mathrm{mV}$ for $30 \mathrm{msec}$ followed by $24-\mathrm{h}$ incubation with serum-free medium.

Immunocytochemistry. LX-2 cells that had been transfected with TM6SF2 expression plasmid or treated with siRNA were incubated for $48 \mathrm{~h}$ were fixed with $4 \%$ (v/v) paraformaldehyde and stained with anti-TM6SF2 antibody. The bound antibodies were detected with an Alexa 594-conjugated antibody against rabbit $\operatorname{IgG}(1: 2,000)$ (Molecular Probes). Nuclei were counterstained with bisbenzimide H 33258 (Hoechst 33258; Abcam). The stained cells were examined using a Fluoview FV10i microscope (Olympus Co.). Fluorescence intensities of TM6SF2 were compared using the Mann-Whitney U test.

Statistical analysis. All experiments were performed in triplicate wells. All data are expressed as the mean \pm standard deviation (SD) and are presented relative to control. Pairwise differences between groups were examined for statistical significance using the Mann-Whitney U test. Univariate or 
A

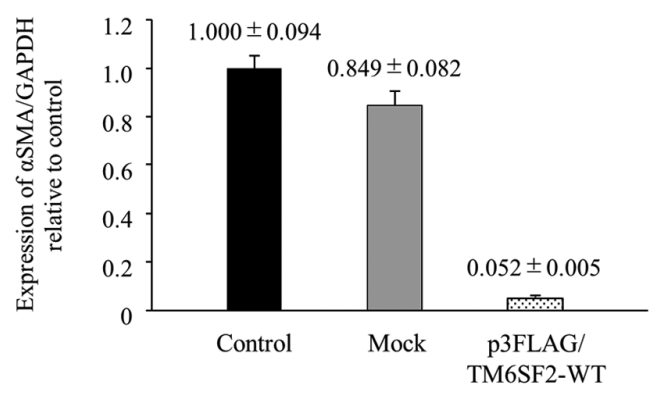

C

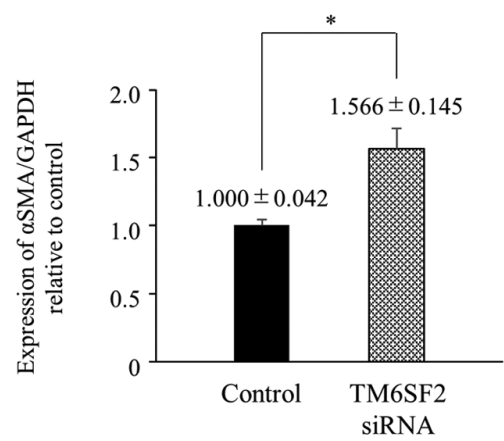

B

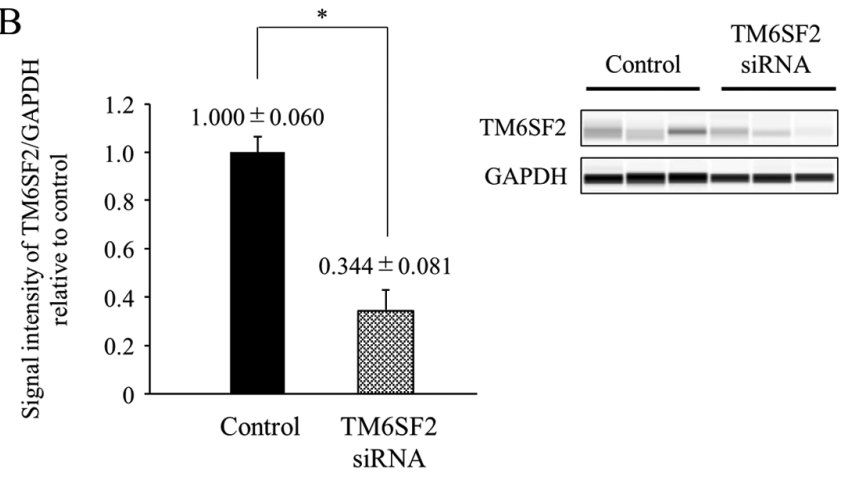

D

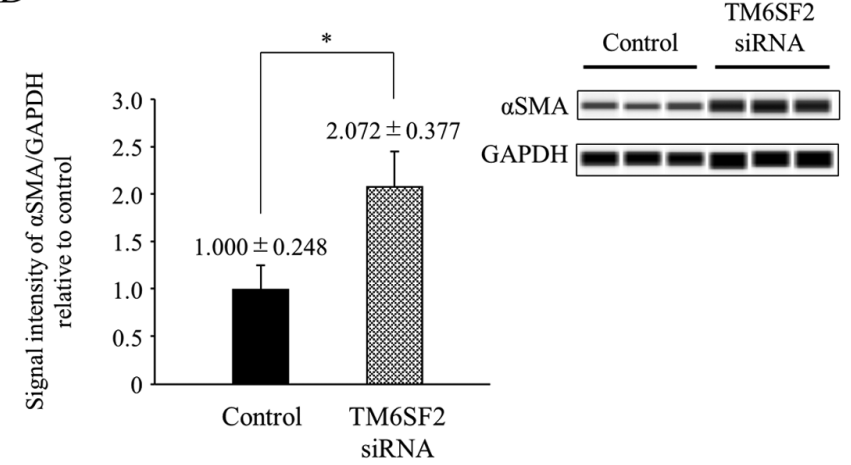

Figure 1. TM6SF2 regulates $\alpha$ SMA expression in LX-2 cells. (A) The cloned TM6SF2 expression plasmid (p3FLAG/TM6SF2-WT) and empty vector (Mock) were transiently transfected into LX-2 cells followed by $24 \mathrm{~h}$ of incubation. Intracellular $\alpha$ SMA expressions were measured by quantitative PCR. The expression of GAPDH served as a control. Experiments were performed in triplicate wells. (B) Non-treated and TM6SF2 knocked-down LX-2 lysates were transferred onto a automated capillary western blot analysis. Anti-TM6SF2 antibody or anti-GAPDH antibody were applied, followed by anti-rabbit immunoglobulin. Signal intensity was corrected by GAPDH and is shown in the bar graph. (C) Intracellular $\alpha$ SMA expression, measured by quantitative PCR, was compared in non-treated and TM6SF2 knocked-down LX-2 cells. GAPDH expression was used as a control. Experiments were performed in triplicate wells. (D) Non-treated and TM6SF2 knocked-down LX-2 lysates were transferred onto an automated capillary western blotting system. Anti- $\alpha$ SMA antibody or anti-GAPDH antibody were applied, followed by anti-rabbit immunoglobulin. Signal intensities were corrected by GAPDH and are presented in the bar graph. Experiments were performed in duplicate wells. ${ }^{*} \mathrm{P}<0.05$. TM6SF2, transmembrane 6 superfamily 2 ; TGF $\beta 1$, transforming growth factor $\beta 1$; $\alpha$ SMA, $\alpha$-smooth muscle actin; si, small interfering.

multivariable differences among three or more groups were estimated using one-way or two-way ANOVA with Tukey's post-hoc multiple comparison test. $\mathrm{P}<0.05$ was considered to indicate a statistically significant difference. Statistical analysis was performed using IBM SPSS Statistics for Windows, version 22.0 (IBM Corp.).

\section{Results}

TM6SF2 regulates $\alpha$ SMA expression in $L X-2$ cells. To analyze the impact of TM6SF2 on HSC activation, p3FLAG/TM6SF2-WT plasmid was transiently transfected into LX-2 cells, and the induction of alpha-smooth muscle actin ( $\alpha$ SMA) level was compared. Although $\alpha$ SMA level was not changed by transfection with empty vector (mock), $\alpha S M A$ mRNA expression was significantly suppressed in the presence of TM6SF2 expression plasmids (one-way ANOVA, $\mathrm{P}<0.05$, respectively) (Fig. 1A).

To verify this result, we also analyzed the association between TM6SF 2 and $\alpha S M A$ by knocking down TM6SF2. The siRNA targeted to TM6SF2 was transfected into LX-2 cells by electroporation, and intracellular $\alpha$ SMA level was compared $24 \mathrm{~h}$ after siRNA treatment. TM6SF2 protein expression in
LX-2 cells was suppressed to $34.4 \%$ by treatment with siRNA (Fig. 1B), and immunostaining of TM6SF2 exhibited the same results (Figs. S1 and 2). $\alpha$ SMA expression in TM6SF2-knocked down cells was 1.5 2.0-fold elevated compared to control cells in both mRNA and protein levels (Fig. 1C and D). A similar tendency was also observed in intracellular collagen type 1 alpha $1(C O L 1 A 1)$ expression measured by real-time PCR (Fig. S3). These results suggest that TM6SF2 downregulates aSMA expression in HSCs.

TM6SF2 suppresses $\alpha$ SMA induction by TGF $\beta 1$ in $L X-2$ cells. To analyze the influence of TM6SF2 on $\alpha$ SMA expression under TGF $\beta 1$ stimulation, changes in $\alpha$ SMA expression in LX-2 cells were compared after TGF $\beta 1$ treatment. LX-2 cells were transfected with p3FLAG/TM6SF2-WT plasmid. Twenty-four hours after transfection, the cells were treated with $10 \mathrm{ng} / \mathrm{ml}$ of TGF $\beta 1$ for $48 \mathrm{~h}$, and intracellular $\alpha$ SMA induction was analyzed by quantitative PCR. $\alpha S M A$ mRNA levels in TM6SF2-overexpressed LX-2 cells were significantly suppressed and failed to increase to control levels following TGF $\beta 1$ stimulation (Fig. 2A). A similar tendency was also observed in the siRNA experiment. The $\alpha$ SMA expression level in TGF $\beta 1$-stimulated LX-2 cells were similar 
A

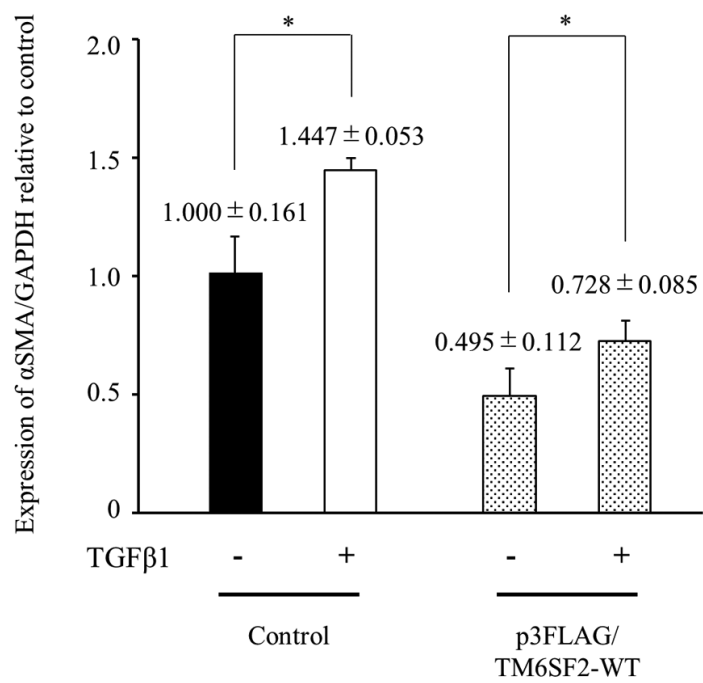

$\mathrm{B}$

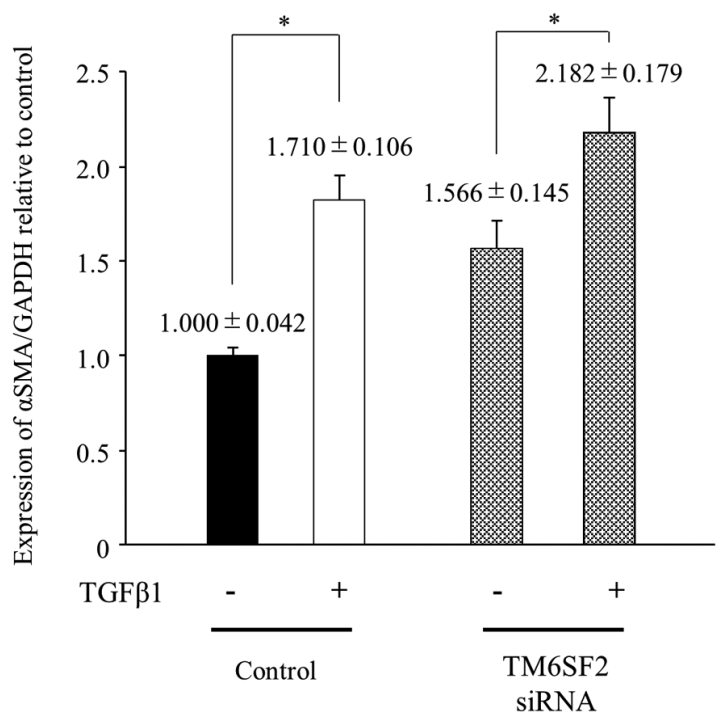

Figure 2. TM6SF2 suppresses $\alpha$ SMA induction by TGF 31 in LX-2 cells. (A) The cloned TM6SF2 expression plasmid (p3FLAG/TM6SF2-MT) and empty vector (Mock) were transiently transfected into LX-2 cells followed by $24 \mathrm{~h}$ of incubation. LX-2 cells were stimulated with or without 10 ng/ml of TGF $\beta 1$ for $48 \mathrm{~h}$. Intracellular $\alpha$ SMA expression was measured using quantitative PCR. The expression of GAPDH served as a control. Experiments were performed in triplicate wells. (B) Non-treated and TM6SF2 knocked-down LX-2 cells were stimulated with or without $10 \mathrm{ng} / \mathrm{ml}$ of TGF $\beta 1$ for $48 \mathrm{~h}$ and intracellular $\alpha \mathrm{SMA}$ expression was compared using quantitative PCR. The expression of GAPDH served as a control. Experiments were performed in triplicate wells. * $<0.05$. TM6SF2, transmembrane 6 superfamily 2; TGF $\beta 1$, transforming growth factor $\beta 1 ; \alpha$ SMA, $\alpha$-smooth muscle actin; MT, mutant type; si, small interfering.

in TM6SF2-knock down LX-2 cells, and the expression of $\alpha$ SMA was enhanced under TGF $\beta 1$ stimulation (Fig. 2B). These results suggested that HSCs could be additionally activated via TGF $\beta 1$ stimulation under lower expression of TM6SF2.

The impact of TM6SF2 phenotype on $\alpha$ SMA induction. To analyze the impact of the substitution at TM6SF2 amino acid 167, $\alpha$ SMA expression was compared between LX-2 cells transfected with p3FLAG/TM6SF2-WT plasmid and with p3FLAG/TM6SF2-MT plasmid. $\alpha S M A$ mRNA expression in cells transfected with p3FLAG/TM6SF2-WT plasmid was lower than that with p3FLAG/TM6SF2-MT plasmid (Fig. 3A; $\mathrm{P}<0.05)$. Similarly, $\alpha \mathrm{SMA}$ protein expression was $>50 \%$ suppressed following transfection with p3FLAG/TM6SF2-WT plasmid compared to transfection with p3FLAG/TM6SF2-MT plasmid (Fig. 3B; P<0.05).

LX-2 cells that had been transfected with or without TM6SF2 expression plasmid were stimulated by TGF $\beta 1$, and intracellular $\alpha$ SMA induction was analyzed by quantitative PCR. Although $\alpha$ SMA mRNA levels were suppressed by TM6SF2 expression, $\alpha$ SMA expression level in TM6SF2 E167K isoform (p3FLAG/TM6SF2-MT)-overexpressed LX-2 cells recovered and reached levels similar to those of control or mock cells after TGF $\beta 1$ stimulation (Fig. 3C). Cell transfection and TGF $\beta 1$ stimulation independently affected $\alpha$ SMA expression in LX-2 cells (two-way ANOVA; $\mathrm{P}<0.05$ ), and, in particular, overexpression of p3FLAG/TM6SF2-WT plasmid significantly affected $\alpha$ SMA expression (Tukey's post-hoc multiple comparison test; $\mathrm{P}<0.05)$. A similar tendency was also observed in intracellular COL1A1 expression estimated by real-time PCR (Fig. S4). These results suggest that basal aSMA expression in HSCs with TM6SF2 wild-type might be low but might be upregulated by TGF $\beta 1$ to a much higher level than HSCs with the TM6SF2 E167K isoform.

\section{Discussion}

NAFLD and NASH are progressive liver diseases characterized by accumulation of fat in human hepatocytes and an increased risk of cirrhosis or hepatocellular carcinoma. The number of patients is increasing worldwide, accompanied by recent upward trends in obesity, westernized high-fat oral intake, gut dysbiosis, inadequate exercise, and comorbid metabolic disorders like diabetes mellitus $(2,24)$. To identify factors associated with NAFLD, clinical studies have concluded that the prevalence, prognosis, and progression or severity of disease is significantly associated with SNPs in PNPLA3 (rs738409) and TM6SF2 (rs58542926) (4,5,11,25,26). Several studies have shown that the rs738409SNP in PNPLA3 causes a loss-of-function amino acid substitution (I148M) in PNPLA3 that affects regulation of lipid droplets in human hepatocytes and retinol metabolism in human HSCs, resulting in positive modulation of HSC activation $(27,28)$. However, the functional impact of the coding SNP in TM6SF 2 has not been sufficiently clarified. Although it has been reported that TM6SF2 is highly expressed in the liver, kidney, brain, and small intestine and that the E167K amino acid substitution TM6SF2 (rs58542926) interferes with localization to the endoplasmic reticulum due to protein misfolding $(14,20)$, the association between the existence of the coding SNP in TM6SF2 and activation of HSCs has not been fully elucidated.

We first analyzed the association between TM6SF2 and the activation of human HSCs. Intracellular $\alpha$ SMA mRNA expression in LX-2 cells was suppressed by TM6SF2 overexpression, and its expression was increased by knocking down TM6SF2 (Fig. 1A and D). Since similar results were observed in the other experiments (Fig. 2A and B), these data suggest that TM6SF2 negatively regulates HSC activation.

In the progression of liver fibrosis, it is well known that TGF $\beta 1$, secreted directly by HSCs or by activated Kupffer cells, 
A

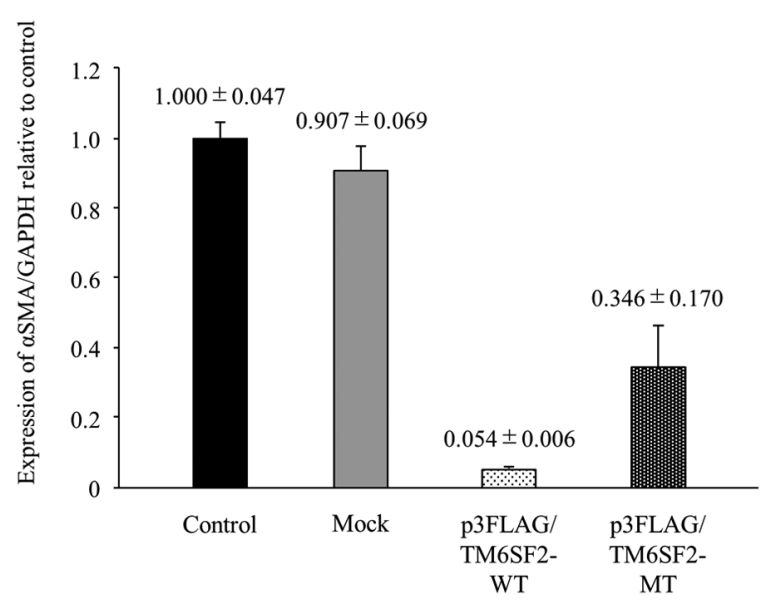

WT $\quad$ MT
$\mathrm{B}$

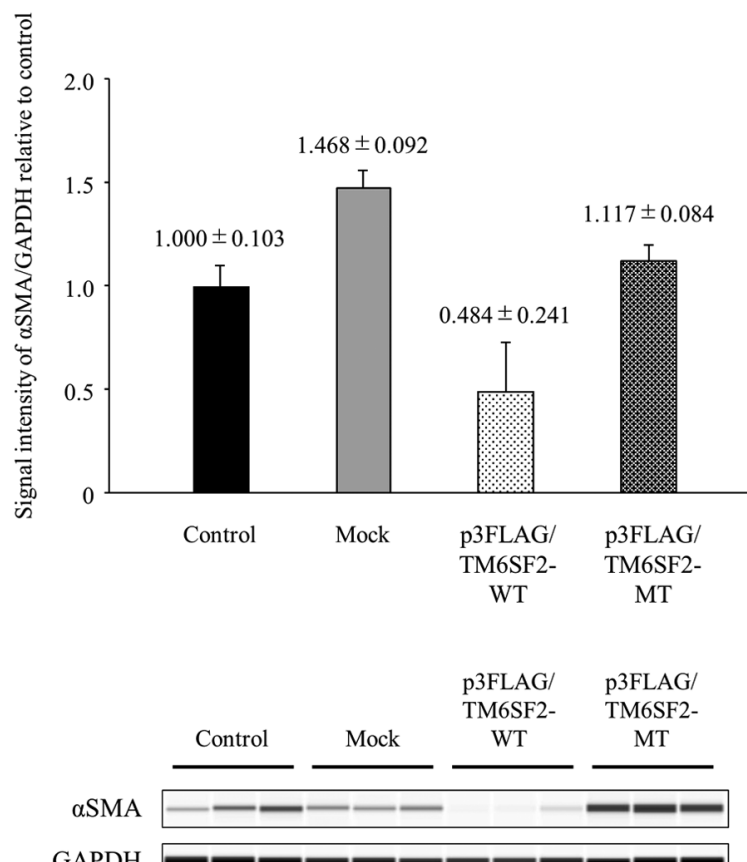

$\mathrm{C}$

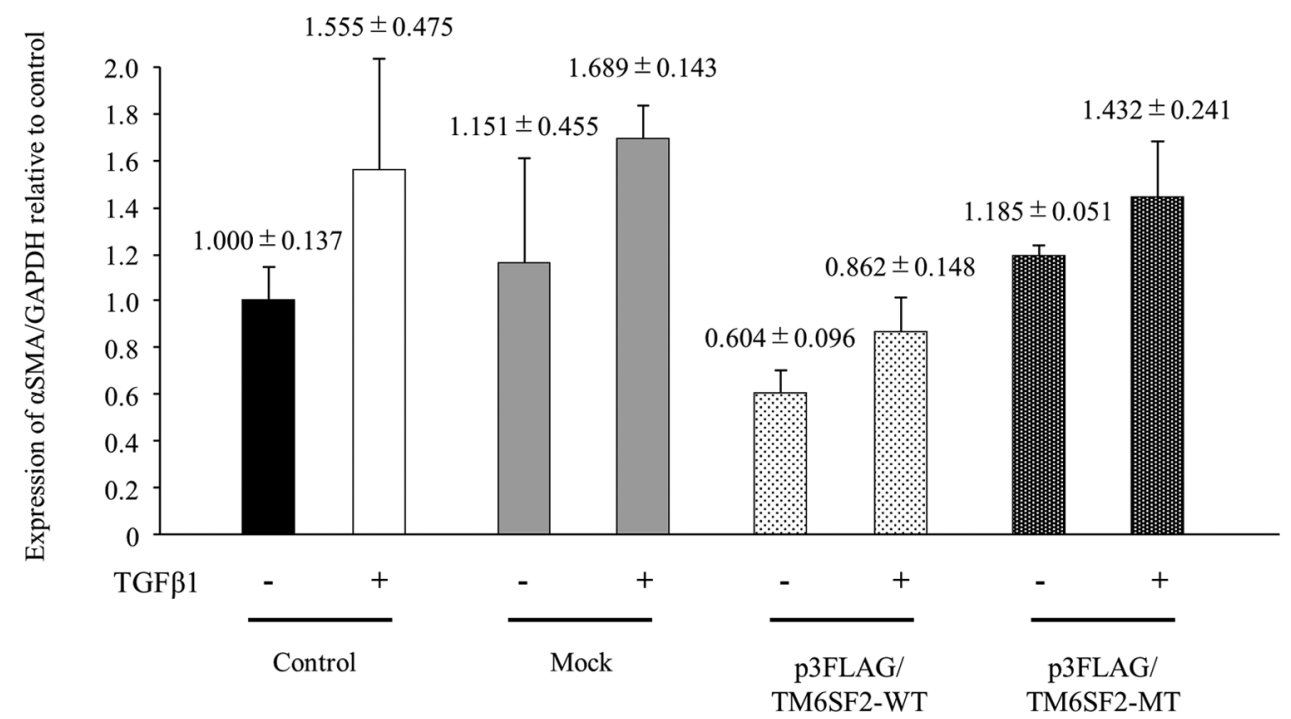

Figure 3. The impact of TM6SF2 phenotype on aSMA induction in LX-2 cells. (A) The cloned TM6SF2 expression plasmid consisting of p3FLAG/TM6SF2-WT and p3FLAG/TM6SF2-MT and empty vector (Mock) were transiently transfected into LX-2 cells, followed by $24 \mathrm{~h}$ of incubation. Intracellular $\alpha$ SMA expression was measured using quantitative PCR, with the expression of GAPDH serving as a control. Experiments were performed in triplicate wells. (B) Cloned TM6SF2 expression plasmids consisting of p3FLAG/TM6SF2-WT and p3FLAG/TM6SF2-MT and empty vector (Mock) were transiently transfected into LX-2 cells, followed by $24 \mathrm{~h}$ of incubation. LX-2 lysates were transferred onto a automated capillary western blotting system. Anti-TM6SF2 antibody or anti-GAPDH antibody were applied, followed by anti-rabbit immunoglobulin. Signal intensity was corrected by GAPDH, as shown in the bar graph. Experiments were performed in triplicate wells. (C) Cloned TM6SF2 expression plasmids consisting of p3FLAG/TM6SF2-WT and p3FLAG/TM6SF2-MT and empty vector (Mock) were transiently transfected into LX-2 cells, followed by $24 \mathrm{~h}$ of incubation. LX-2 cells were stimulated with or without $10 \mathrm{ng} / \mathrm{ml}$ of TGF $\beta 1$ for $48 \mathrm{~h}$. Intracellular $\alpha$ SMA expression was measured using quantitative PCR, with GAPDH as a control. Experiments were performed in triplicate wells. TM6SF2, transmembrane 6 superfamily 2; TGF $\beta 1$, transforming growth factor $\beta 1$; $\alpha$ SMA, $\alpha$-smooth muscle actin; MT, mutant type; WT, wild-type.

could activate HSCs, triggering transformation of HSCs to myofibroblasts (22). Thus, we analyzed the impact of TM6SF2 on HSC activation via TGF $\beta 1$ signaling. Although intracellular $\alpha$ SMA expression in both control cells and TM6SF2 over-expressed cells was significantly upregulated by TGF $\beta 1$ treatment, $\alpha$ SMA expression in TM6SF2 overexpressed LX-2 cells was significantly lower than that in control LX-2 cells after TGF $\beta 1$ treatment (Fig. 2A). Similar results were observed in TM6SF2 knock down cells (Fig. 2B). A similar tendency was also observed in intracellular COL1A1 expression measured using real time PCR (Figs. S3 and 4). These results indicate that lower TM6SF2 expression could activate HSCs and that TGF $\beta 1$ could enhance this HSC activation.

Subsequently, we analyzed the functional impact of the coding SNP in TM6SF2 in vitro. Normal HSCs have lipid droplets containing retinol. However, once HSCs are activated, lipid droplets are diminished, and retinyl ester is degraded in the endoplasmic reticulum (ER) in HSCs (29). Since the 
amino acid substitution (E167K) in TM6SF2 (rs58542926 SNP) causes TM6SF2 to fail to localize to the ER $(18,19)$, we propose that amino acid substitution E167K in TM6SF2 could induce HSC activation by disrupting homeostasis in the ER. When TM6SF2 wild-type or mutant type (E167K) were overexpressed in LX-2 cells, intracellular $\alpha$ SMA in LX-2 cells that overexpressed wild-type TM6SF2 decreased more than those that overexpressed mutant TM6SF2 (Fig. 3). Furthermore, aSMA expression in TM6SF2-mutant-overexpressed LX-2 cells increased to similar levels as control LX-2 cells after treatment with TGF $\beta 1$. Although the precise regulation of TM6SF2 in HSCs could not be determined in this study, our results suggest that the TM6SF2 E167K isoform affects HSC sensitivity by enhancing the response to TGF $\beta 1$.

In the present study, we demonstrated the impact of an amino acid substitution in TM6SF2 on liver fibrosis using LX-2 cells. Although the impact of this TM6SF2 coding SNP on liver fibrosis might not be strong considering the hazard ratio calculated for this SNP by GWAS studies, we consider that these results could help to clarify the role of TM6SF2 and the impact of the TM6SF2 SNP on the progression of liver fibrosis in NAFLD and NASH patients.

TM6SF2 negatively affects $\alpha$ SMA expression in HSCs, and the TM6SF2 E167K isoform associated with the $r s 58542926$ SNP might affect HSC activation sensitivity. These results suggest that TM6SF2 might play a role in the process of HSC activation and liver fibrosis in NASH.

\section{Acknowledgements}

Not applicable.

\section{Funding}

The present study was supported by grants-in-aid for scientific research and development from the Ministry of Health, Labor and Welfare and Ministry of Education Culture Sports Science and Technology (JSPS KAKENHI grant no. 18K15814) and Japan Agency for Medical Research and Development (grant no. JP20fk0210040). The present study was also supported by the Gilead Sciences Research Scholars Program in Liver Disease by Gilead Sciences, Inc. The funders had no role in study design, data collection and analysis, decision to publish, or preparation of the manuscript.

\section{Availability of data and materials}

The datasets used and/or analyzed during the current study are available from the corresponding author on reasonable request.

\section{Authors' contributions}

SL, EM and KC conceived the study. GNM, AO, DM and HAC made contributions to the design of the experiment. SL and EM analyzed and interpreted the experimental data. TN, KO, YT, TU, KM, HF, MY, TK and AH were involved in analyzing the data and revising the manuscript. MT performed western blotting experiments and edited the manuscript. DM and AO performed part of the immunostaining experiments and checked gene expression analysis data. MI and HA designed the study and confirmed the quality of experimental data. $\mathrm{CNH}$ contributed to statistical analysis and proofreading. MT and $\mathrm{CNH}$ were major contributors in editing the manuscript. $\mathrm{KC}$ revised the manuscript and gave the final approval of the version to be submitted. All authors read and approved the final manuscript.

\section{Ethics approval and consent to participate}

Not applicable.

\section{Patient consent for publication}

Not applicable.

\section{Competing interests}

The authors declare that they have no competing interests.

\section{References}

1. Eslam M, Valenti L and Romeo S: Genetics and epigenetics of NAFLD and NASH: Clinical impact. J Hepatol 68: 268-279, 2018.

2. Kleiner DE, Brunt EM, Van Natta M, Behling C, Contos MJ, Cummings OW, Ferrell LD, Liu YC, Torbenson MS, Unalp-Arida A, et al: Design and validation of a histological scoring system for nonalcoholic fatty liver disease. Hepatology 41 : 1313-1321, 2005

3. Angulo P, Kleiner DE, Dam-Larsen S, Adams LA, Bjornsson ES, Charatcharoenwitthaya P, Mills PR, Keach JC, Lafferty HD, Stahler A, et al: Liver fibrosis, but no other histologic features, is associated with long-term outcomes of patients with nonalcoholic fatty liver disease. Gastroenterology 149: 389-397, 2015.

4. Romeo S, Kozlitina J, Xing C, Pertsemlidis A, Cox D, Pennacchio LA, Boerwinkle E, Cohen JC and Hobbs HH: Genetic variation in PNPLA3 confers susceptibility to nonalcoholic fatty liver disease. Nat Genet 40: 1461-1465, 2008.

5. Speliotes EK, Yerges-Armstrong LM, Wu J, Hernaez R, Kim LJ, Palmer CD, Gudnason V, Eiriksdottir G, Garcia ME, Launer LJ, et al: Genome-wide association analysis identifies variants associated with nonalcoholic fatty liver disease that have distinct effects on metabolic traits. PLoS Genet 7: e1001324, 2011.

6. Singal AG, Manjunath H, Yopp AC, Beg MS, Marrero JA, Gopal P and Waljee AK: The effect of PNPLA3 on fibrosis progression and development of hepatocellular carcinoma: A meta-analysis. Am J Gastroenterol 109: 325-334, 2014.

7. Hotta K, Yoneda M, Hyogo H, Ochi H, Mizusawa S, Ueno T, Chayama K, Nakajima A, Nakao K and Sekine A: Association of the rs738409 polymorphism in PNPLA3 with liver damage and the development of nonalcoholic fatty liver disease. BMC Med Genet 11: 172, 2010.

8. Sookoian S, Castaño GO, Scian R, Mallardi P, Fernández Gianotti T, Burgueño AL, San Martino J and Pirola CJ: Genetic variation in transmembrane 6 superfamily member 2 and the risk of nonalcoholic fatty liver disease and histological disease severity. Hepatology 61: 515-525, 2015.

9. Pirola CJ and Sookoian SL: The dual and opposite role of the TM6SF2-rs58542926 variant in protecting against cardiovascular disease and conferring risk for nonalcoholic fatty liver: A meta-analysis. Hepatology 62: 1742-1756, 2015.

10. Macaluso FS, Maida M and Petta S: Genetic background in nonalcoholic fatty liver disease: A comprehensive review. World J Gastroenterol 21: 11088-11111, 2015.

11. Goffredo M, Caprio S, Feldstein AE, D'Adamo E, Shaw MM, Pierpont B, Savoye M, Zhao H, Bale AE and Santoro N: Role of TM6SF2 rs58542926 in the pathogenesis of nonalcoholic pediatric fatty liver disease: A multiethnic study. Hepatology 63: 117-125, 2016. 
12. Liu YL, Reeves HL, Burt AD, Tiniakos D, McPherson S, Leathart JB, Allison ME, Alexander GJ, Piguet AC, Anty R, et al: TM6SF2 rs58542926 influences hepatic fibrosis progression in patients with Non-alcoholic fatty liver disease. Nat Commun 5: 4309, 2014

13. Dongiovanni P, Petta S, Maglio C, Fracanzani AL, Pipitone R, Mozzi E, Motta BM, Kaminska D, Rametta R, Grimaudo S, et al: Transmembrane 6 superfamily member 2 gene variant disentangles nonalcoholic steatohepatitis from cardiovascular disease. Hepatology 61: 506-514, 2015.

14. Li TT, Li TH, Peng J, He B, Liu LS, Wei DH, Jiang ZS, Zheng XL and Tang ZH: TM6SF2: A novel target for plasma lipid regulation. Atherosclerosis 268: 170-176, 2018.

15. O'Hare EA, Yang R, Yerges-Armstrong LM, Sreenivasan U, McFarland R, Leitch CC, Wilson MH, Narina S, Gorden A, Ryan KA, et al: TM6SF2 rs58542926 impacts lipid processing in liver and small intestine. Hepatology 65: 1526-1542, 2017.

16. Luukkonen PK, Zhou Y, Nidhina Haridas PA, Dwivedi OP, Hyötyläinen T, Ali A, Juuti A, Leivonen M, Tukiainen T, Ahonen L, et al: Impaired hepatic lipid synthesis from polyunsaturated fatty acids in TM6SF2 E167K variant carriers with NAFLD. J Hepatol 67: 128-136, 2017.

17. Prill S, Caddeo A, Baselli G, Jamialahmadi O, Dongiovanni P, Rametta R, Kanebratt KP,Pujia A, Pingitore P, Mancina RM, et al: The TM6SF2 E167K genetic variant induces lipid biosynthesis and reduces apolipoprotein B secretion in human hepatic 3D spheroids. Sci Rep 9: 11585, 2019.

18. Mahdessian H, Taxiarchis A, Popov S, Silveira A, Franco-Cereceda A, Hamsten A, Eriksson P and van't Hooft F: TM6SF2 is a regulator of liver fat metabolism influencing triglyceride secretion and hepatic lipid droplet content. Proc Natl Acad Sci USA 111: 8913-8918, 2014.

19. Smagris E, Gilyard S, BusuRay S, Cohen JC and Hobbs HH: Inactivation of TM6SF2, a gene defective in fatty liver disease, impairs lipidation but not secretion of very low density lipoproteins. J Biol Chem 291: 10659-10676, 2016.

20. Kozlitina J, Smagris E, Stender S, Nordestgaard BG, Zhou HH Tybjærg-Hansen A, Vogt TF, Hobbs $\mathrm{HH}$ and Cohen JC: Exome-wide association study identifies a TM6SF2 variant that confers susceptibility to nonalcoholic fatty liver disease. Nat Genet 46: 352-356, 2014.

21. Li JT, Liao ZX, Ping J, Xu D and Wang H: Molecular mechanism of hepatic stellate cell activation and antifibrotic therapeutic strategies. J Gastroenterol 43: 419-428, 2008.
22. Dewidar B, Mayer C, Dooley S and Meindl-Beinker AN: TGF- $\beta$ in hepatic stellate cell activation and liver fibrogenesis-updated 2019. Cells 8: 1419, 2019.

23. Carpino G, Morini S, Corradini G, Corradini S, Franchitto A, Merli M, Siciliano M, Gentili F, Onetti Muda A, Berloco P, et al: Alpha-SMA expression in hepatic stellate cells and quantitative analysis of hepatic fibrosis in cirrhosis and in recurrent chronic hepatitis after liver transplantation. Dig Liver Dis 37: 349-356, 2005.

24. Henao-Mejia J, Elinav E, Jin C, Hao L, Mehal WZ, Strowig T, Thaiss CA, Kau AL, Eisenbarth SC, Jurczak MJ, et al: Inflammasome-mediated dysbiosis regulates progression of NAFLD and obesity. Nature 482: 179-185, 2012.

25. Eslam M, Mangia A, Berg T, Chan HL, Irving WL, Dore GJ, Abate ML, Bugianesi E, Adams LA, Najim MA, et al: Diverse impacts of the rs58542926 E167K variant in TM6SF2 on viral and metabolic liver disease phenotypes. Hepatology 64: 34-46, 2016.

26. Sookoian S and Pirola CJ: Meta-analysis of the influence of TM6SF2 E167K variant on plasma concentration of aminotransferases across different populations and diverse liver phenotypes. Sci Rep 6: 27718, 2016.

27. Pingitore P, Dongiovanni P, Motta BM, Meroni M, Lepore SM, Mancina RM, Pelusi S, Russo C, Caddeo A, Rossi G, et al: PNPLA3 overexpression results in reduction of proteins predisposing to fibrosis. Hum Mol Genet 25: 5212-5122, 2016.

28. Bruschi FV, Claudel T, Tardelli M, Caligiuri A, Stulnig TM, Marra F and Trauner M: The PNPLA3 I148M variant modulates the fibrogenic phenotype of human hepatic stellate cells. Hepatology 65: 1875-1890, 2017.

29. Testerink N, Ajat M, Houweling M, Brouwers JF, Pully VV, van Manen HJ, Otto C, Helms JB and Vaandrager AB: Replacement of retinyl esters by polyunsaturated triacylglycerol species in lipid droplets of hepatic stellate cells during activation. PLoS One 7: e34945K, 2012.

(i) $(-)$ This work is licensed under a Creative Commons Attribution-NonCommercial-NoDerivatives 4.0 International (CC BY-NC-ND 4.0) License. 